\title{
Factors affecting yield and gelling properties of agar
}

\begin{abstract}
Agar, a gelatinous polysaccharide in the cell wall of many red algal species, is widely used as a gelling, thickening and stabilizing agent. The commercial value of seaweed is judged by their agar content and gel quality. Seaweed materials with higher agar yield and better gelling properties are desired due to the growing demand for agar in the global market. Agar biosynthesis in seaweeds is affected by genetic variations, developmental stages and environmental conditions, while different agar extraction techniques can also affect the yield and quality of agar. In this paper, the effects of different physiological states of seaweed, abiotic and biotic factors, seaweed storage and agar extraction techniques on the agar yield and gelling characteristics, are reviewed. This information is important as a guide for marine aquaculture of potential agarophytes and the possible effects of climate change on the stock of this natural resource.
\end{abstract}

Keyword: Agar; Agarophyte; Agar yield; Cultivation; Gel strength; Gracilaria 